\title{
PROPOSTA DE MELHORIA NO LAYOUT DE UM LABORATÓRIO DE ANÁLISES CLÍNICAS UTILIZANDO O FLUXOGRAMA DE PROCESSO E O DIAGRAMA DE SPAGHETTI
}

\section{PROPOSAL FOR IMPROVEMENT IN THE LAYOUT OF A CLINICAL ANALYSIS LABORATORY USING THE PROCESS FLOWCHART AND THE SPAGHETTI DIAGRAM}

\section{E. L. VIEIRA ${ }^{1}$}

${ }^{1}$ Pontifícia Universidade Católica do Paraná, Departamento de Engenharia de Produção, Curitiba, Paraná, Brasil

${ }^{*}$ Autor Correspondente. Pontifícia Universidade Católica do Paraná, Departamento de Engenharia de Produção, Curitiba, Paraná, Brasil, Phone: +55413271 1513

Endereço de e-mail: vieiraeverton@gmail.com (E. L.Vieira).

\begin{tabular}{l} 
A R T I C L E I N F O \\
\hline Article history: \\
Received 2019-11-26 \\
Accepted 2020-01-03 \\
Available online 2020-02-15 \\
pa lavras-chave \\
Layout \\
Diagrama de Spaghetti \\
Fluxograma de Processo \\
Desperdícios \\
ke ywo r d s \\
Layout \\
Spaghetti chart \\
Process Flow \\
Wastes
\end{tabular}

\begin{abstract}
R E S U M O
Este estudo teve origem na necessidade de um laboratório de análises clínicas em priorizar e aperfeiçoar sua vantagem competitiva. Para isso foram utilizadas as ferramentas de fluxograma de processos e diagrama de spaghetti para estudar o melhor layout para o processo de coleta de exames de sangue, visando a redução de desperdícios. Foram realizados mapeamentos dos processos e identificação dos atores envolvidos para desenho da situação atual, esse levantamento foi realizado através de observações e com uso de fluxograma de processo e diagrama de spaghetti, onde foram identificadas as atividades que agregam valor $(A V)$ e não agregam valor (NAV). Após isso foram propostas melhorias no layout para redução das movimentações realizadas no processo. Com essas propostas foi possível reduzir 45 metros de deslocamento da função do laboratorista, também a redução de $100 \%$ das atividades que não agregam valor da recepcionista. $O$ uso dessas ferramentas foi muito útil para identificação e eliminação dos desperdícios do processo na empresa estudada.
\end{abstract}

A B S T R A C T

This study originated in the need of a clinical analysis laboratory to prioritize and improve its competitive advantage. For this, we used the process flowchart and spaghetti diagram tools to study the best layout for the blood collection process, aiming at the reduction of waste. Processes mapping and identification of the actors involved in the design of the current situation were carried out. This survey was carried out through observations and using a process flowchart and spaghetti diagram, which identified activities that add value (AV) and do not add value (NAV). After this, improvements were proposed in the layout to reduce the movements carried out in the process. With these proposals it was possible to reduce 45 meters of displacement of the function of the laboratory, also the reduction of $100 \%$ of activities that do not add value of the receptionist. The use of these tools was very useful for identifying and eliminating the waste of the process in the company studied. 


\section{INTRODUÇÃO}

Dentro das empresas os processos e atividades são os meios de agregação de valor aos produtos e serviços para atendimento aos clientes, realizando o gerenciamento destes processos, com a finalidade de melhora-los, necessita uma avaliação crítica das práticas da organização produtiva como um todo. (CORRÉA et al, 2001). De acordo com Johansson (1995) realizar o mapeamento de processos, seja qual for a técnica empregada, e uma ferramenta de visualização completa e de compreensão imediata das atividades executadas, tornando-se a estrutura básica para a análise dos pontos possíveis de melhorar a fim de reduzir os recursos empregados nos processos.

O mapeamento de processos com as ferramentas de fluxograma de processos e diagrama de spaghetti auxiliam o desenvolvimento do layout nas empresas. Segundo Peinado e Graeml (2007) o layout e a parte mais visível e exposta de qualquer empresa e a necessidade de estuda-lo existe sempre que se pretende a implantação de um novo processo ou quando estiver promovendo a reformulação do layout atual das empresas.

O objetivo deste estudo de caso e realizar o mapeamento dos processos de um laboratório de análises clínicas para a atividade de coleta de exames de sangue, utilizando o layout da empresa, fluxograma de processos e diagrama de spaghetti para identificação das atividades que agregam valor e não agregam valor, após isso propor melhorias ao processo.

\section{LAYOUT}

Segundo Camarotto e Menegon (2006) o layout pode ser definido de forma simplificada e por definições como: "O layout e a representação espacial dos fatores que concorrem para a produção envolvendo homens, equipamentos, materiais e suas interações.

Slack et al (2009) cita que o layout se preocupa com a localização física dos recursos de transformação, ou seja, seria decidir onde colocar todas as instalações, máquinas, equipamentos e pessoal envolvidos no processo. De acordo com Vieira (2017) um bom layout permite que mão-de-obra, materiais e informações tenham um fluxo eficiente e seguro durante a execução dos processos nas empresas.

\subsection{Diagrama de Spaghetti}

De acordo com Womack e Jones (1998) o diagrama de Spaghetti serve para ilustrar os desperdícios relativos ao deslocamento dos funcionários, pois trata-se de uma ferramenta que mostra o deslocamento de um produto ou o deslocamento de um operador. O diagrama de Spaghetti proporciona a identificação de movimentos desnecessários e o planejamento do layout futuro (MARIZ e PICCHI 2013).

\subsection{Fluxograma de Processo}

De acordo com Peinado e Graeml (2007), os fluxogramas são formas de representar por meio de símbolos gráficos, a sequência das etapas de um trabalho para facilitar sua análise. Barnes (1977) cita que o mapeamento de processos representa os diversos passos ou eventos que ocorrem durante a execução de uma tarefa específica, ou durante uma série de ações. Os símbolos utilizados para fluxogramas de processos são representados na figura 1.

\begin{tabular}{|l|l|}
\hline SÍMBOLO & \multicolumn{1}{|c|}{ DESCRIÇÃo } \\
\hline & $\begin{array}{l}\text { Operação: Ocorre quando se modifica } \\
\text { intencionalmente um objeto em qualquer de suas } \\
\text { caracteristicas fisicas ou quimicas, ou também } \\
\text { quando se monta ou desmonta componentes ou } \\
\text { partes. }\end{array}$ \\
\hline & $\begin{array}{l}\text { Transporte: Ocorre quando um objeto ou matéria } \\
\text { prima é transferido de um lugar para o outro, de uma } \\
\text { seção para outra, de um prédio para outro. }\end{array}$ \\
\hline & $\begin{array}{l}\text { Espera ou demora: Ocorre quando um objeto ou } \\
\text { matéria prima é colocado intencionalmente numa } \\
\text { posição estática. o material permanece aguardando } \\
\text { processamento ou encaminhamento. }\end{array}$ \\
\hline & $\begin{array}{l}\text { Inspeçäo: Ocorre quando um objeto ou matéria prima } \\
\text { é examinado para sua identificação, quantidade ou } \\
\text { condição de qualidade. }\end{array}$ \\
\hline & $\begin{array}{l}\text { Armazenagem: Ocorre quando um objeto ou matéria } \\
\text { prima é mantido em área protegida especifica na } \\
\text { forma de estoque. }\end{array}$ \\
\hline
\end{tabular}

Figura 1 - Simbologia Fluxograma de Processos (Adaptado de Peinado e Graeml (2007)

Em geral, o mapeamento das atividades pode melhorar o foco da organização no cliente, auxiliar na eliminação das operações que agregam valor ao produto e reduzir a complexidade do processo (AGUIAR E WESTON, 1993). Para Barnes (1982) existem quatro enfoques principais que se devem considerar diante o desenvolvimento a partir do mapeamento de processos: a eliminação de todo trabalho desnecessário, a combinação de operações ou elementos, a modificação das sequências das operações e a simplificação das operações essenciais.

\subsection{Manufatura Enxuta}

Segundo Shingo (1996) o conceito do pensamento enxuto é a eliminação dos desperdícios dentro das empresas. Ohno (1997) cita que desperdício se refere a todos os elementos de produção que só aumentam os custos sem agregar valor, ou seja, são atividades que não agregam valor ao produto, do ponto de vista do cliente, mas são realizadas dentro dos processos nas empresas.

Shingo (1981) considera que os sete desperdícios para o Sistema Toyota de Produção são:

- Espera: longos períodos de ociosidade de peças, pessoas e informações, resultando em um fluxo pobre, bem como em lead times longos.

- Produção em excesso: produzir excessivamente ou cedo demais, resultando em um fluxo pobre de peças e informações ou excesso de estoques.

- Transporte: movimento excessivo de pessoas, informações ou peças, resultando em disponibilidade desnecessária de capital, tempo e energia.

- Processamento em excesso: utilização do jogo errado de ferramentas, sistemas ou procedimentos, geralmente quando uma aproximação mais simples pode ser mais efetiva.

- Estoques: armazenamento excessivo e falta de informações ou produtos, resultando em custos excessivos e baixo desempenho do serviço prestado ao cliente. 
- Movimentação em excesso: desorganização do ambiente de trabalho, resultando em baixo desempenho dos aspectos ergonômicos e perda frequente de itens.

- Defeito: problemas frequentes de qualidade nos produtos ou baixo desempenho na entrega.

Todos estes desperdícios acabam gerando atividades que não agregam valor aos processos, fazendo com que as empresas gastem muitos recursos por não saberem identifica-los e eliminá-los.

\section{METODOLOGIA}

Com a intenção de esclarecer a situação atual do laboratório e buscar melhorias para alavancar a produtividade, foi inicialmente elaborado o fluxograma de processo da situação atual dos 03 atores envolvidos no processo, que são os clientes, laboratoristas e recepcionista. Para isso, foram executados os seguintes procedimentos: Selecionar o tipo de exame que possui um volume maior de serviços, que no caso foi o exame de sangue, desenhar o processo atual através do fluxograma de processos e diagrama de spaghetti, diagnóstico dos desperdícios, elaboração de mapeamento com melhorias propostas, diagrama de spaghetti e layout adaptado.

Para representar o fluxo das atividades do processo de coleta e análise de exames de sangue, foi necessário seguir o caminho percorrido pelos atores envolvidos no processo, levantamento de distâncias percorridas e atividades que agregam ou não agregam valor ao processo. O Fluxograma de processo foi elaborado com a utilização do software Excel®. Para o desenvolvimento do mesmo, foi necessário a observação de perto de todos os processos, com coleta de informações in loco. O diagrama de Spaghetti foi desenvolvido com auxílio do software Autocad $\AA$, onde o layout atual e o futuro foram desenhados.

Para identificação dos desperdícios presentes na coleta de exames de sangue foi realizada análise crítica sobre todo o fluxo de execução do serviço, baseando-se nos sete tipos de perdas previstos pelo pensamento enxuto.

Por fim, foram determinadas as causas dos desperdícios e elaborado um fluxograma de processo, diagrama de spaghetti e layout com as melhorias propostas. Foram sugeridas melhorias para o laboratório ao final do trabalho.

\section{RESULTADOS}

\subsection{Situação Atual}

O laboratório de análises clínicas realiza atendimentos a pacientes que necessitam realizar a coleta de exames de sangue, urina, fezes, toxicológico, etc. O processo avaliado foi o de coleta de exames de sangue, pois representa o produto com maior volume de atendimentos. Este processo possui três atores que participam diretamente das atividades: recepcionista, paciente e laboratorista. As atividades do laboratorista estão descritas na figura 2 através de um fluxograma de processo.

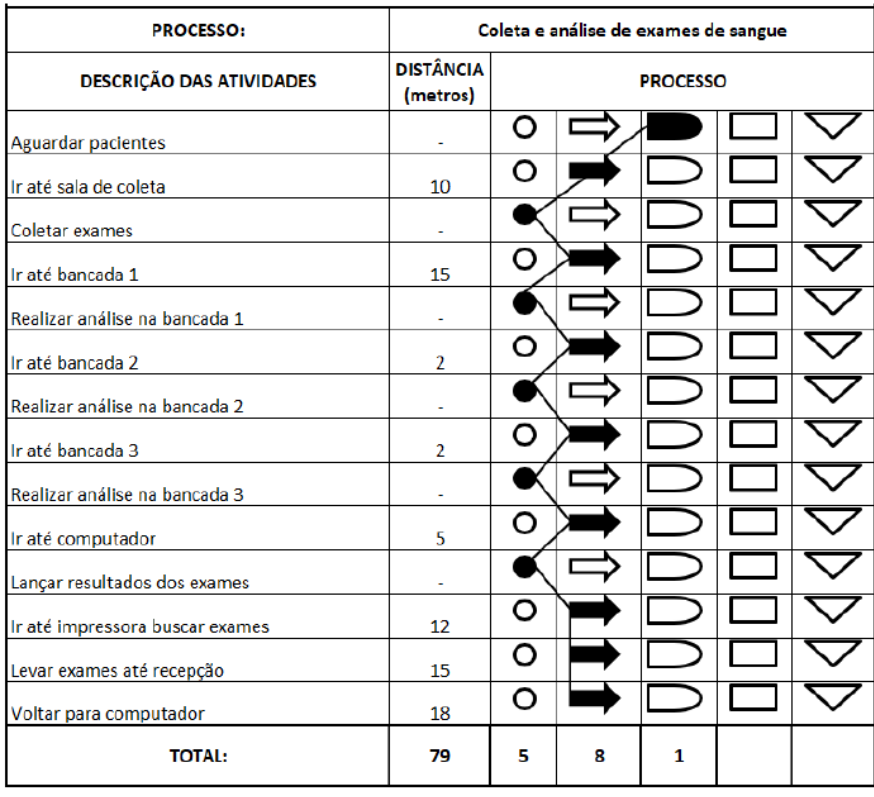

Figura 2 - Fluxograma de processo laboratorista situação atual

A figura 2 descreve as atividades realizadas pelo laboratorista para o processo de coleta de sangue, nota-se que a distância percorrida para essa atividade e de 79 metros, possui 5 operações, 8 transportes e 1 espera.

Para o ator recepcionista também foi desenvolvido um Fluxograma de processo, que pode ser visualizado na figura 3.

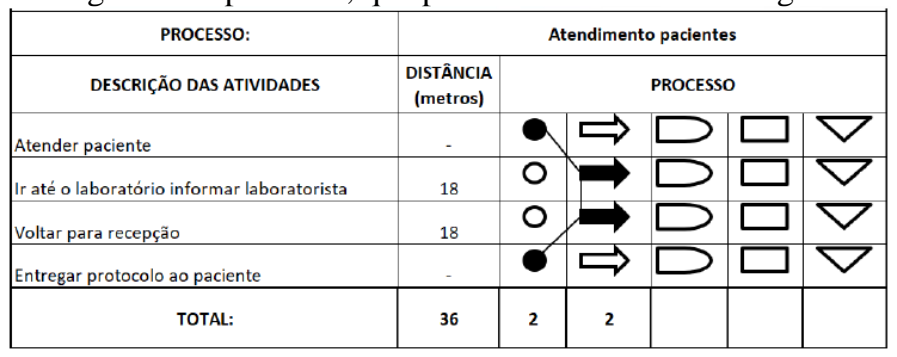

Figura 3 - Fluxograma de processo recepcionista situação atual

As atividades realizadas pela recepcionista consistem em 2 operações e 2 transportes, totalizando 36 metros percorridos nos deslocamentos.

A figura 4 mostra as atividades realizadas pelos pacientes no processo de coleta de exames de sangue. 


\begin{tabular}{|c|c|c|c|c|c|c|}
\hline PROCESSO: & \multicolumn{5}{|c|}{ Coleta sangue paciente } & \\
\hline DESCRIÇÃO DAS ATIVIDADES & $\begin{array}{c}\text { DISTÂNCIA } \\
\text { (metros) }\end{array}$ & & & PROCESS & & \\
\hline Chegar no laboratório e ir até recepção & 5 & 0 & & $\square$ & $\square$ & \\
\hline Apresentar-se para recepcionista & - & & & $\square$ & $\square$ & \\
\hline Ir até sala de coleta & 10 & 0 & & D & $\square$ & \\
\hline Realizar a coleta & - & & & $\square$ & $\square$ & \\
\hline Ir até recepção retirar protocolo & 10 & 0 & & $\square$ & $\square$ & $\checkmark$ \\
\hline Sair do laboratório & 5 & 0 & & $\square$ & $\square$ & V \\
\hline TOTAL: & 30 & 2 & 4 & & & \\
\hline
\end{tabular}

Figura 4 - Fluxograma de processo paciente situação atual

As atividades realizadas pelo paciente consistem em 2 operações e 4 transportes, totalizando 25 metros percorridos nos deslocamentos.

O layout do laboratório foi desenhado para analisar a disposição dos setores, juntamente com o diagrama de spaghetti para verificar as movimentações realizadas por cada ator do processo, conforme figura 5.

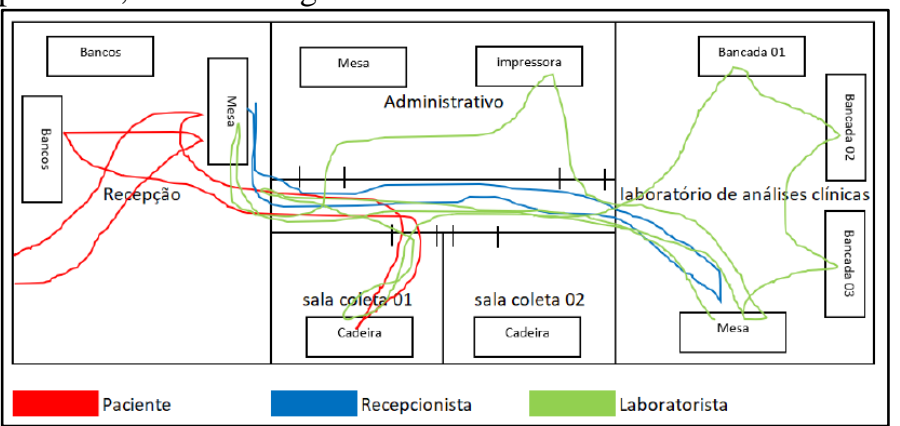

Figura 5 - Layout atual do processo e diagrama de spaghetti

Nota-se que existem muitas atividades que não agregam valor (NAV) no processo, principalmente no que diz respeito a movimentações e transportes para cada ator envolvido, conforme a tabela 1 .

\begin{tabular}{l|c|c|c}
\hline \multicolumn{1}{c|}{ Atores } & \% Atividades AV & \% Atividades NAV & $\begin{array}{c}\text { Distância } \\
\text { percorrida (metros) }\end{array}$ \\
\hline Laboratorista & $35,7 \%$ & $64,4 \%$ & 79 \\
\hline Recepcionista & $50,0 \%$ & $50,0 \%$ & 36 \\
\hline Paciente & $33,3 \%$ & $66,7 \%$ & 30 \\
\hline
\end{tabular}

\section{Tabela 1 - Atividades AV, NAV e distâncias percorridas}

Verificou-se que o laboratorista e o ator do processo que mais gera desperdícios, tendo apenas 35,7\% de agregação de valor nas suas atividades, pois e o que realiza mais movimentações na execução das suas atividades, totalizando 79 metros.

\subsection{Situação Proposta}

Para analisar o processo, foi realizada uma reunião informal com os colaboradores do laboratório, onde foi possível visualizar a situação atual do processo e os desperdícios existentes durante a execução das atividades. Foram sugeridas algumas práticas de gestão visual e organização das atividades, que serão descritas na sequência. Para o laboratorista foi desenhado um novo fluxograma de processo com as propostas de melhoria, conforme figura 6.

\begin{tabular}{|c|c|c|c|c|c|c|}
\hline PROCESSO: & \multicolumn{6}{|c|}{ Coleta e análise exame de sangue } \\
\hline DESCRIÇÃO DAS ATIVIDADES & $\begin{array}{c}\text { DISTÂNCIA } \\
\text { (metros) }\end{array}$ & \multicolumn{5}{|c|}{ PROCESSO } \\
\hline Aguardar paciente & - & $\mathrm{O}$ & & & & \\
\hline Ir até sala de coleta & 10 & & & & & \\
\hline Coletar exames & - & & & & & \\
\hline Ir até bancada 1 & 15 & & & & & \\
\hline Realizar análise na bancada 1 & - & & & & & \\
\hline Ir até bancada 2 & 2 & & & & & \\
\hline Realizar análise na bancada 2 & - & & & & & \\
\hline Ir até bancada 3 & 2 & & & & & \\
\hline Realizar análise na bancada 3 & - & & & & & \\
\hline Ir até computador & 5 & & & & & \\
\hline Lançar exames e mandar imprimir na recepçăo & - & & $\vec{\partial}$ & P & & \\
\hline TOTAL: & 34 & 5 & 6 & 1 & & \\
\hline
\end{tabular}

\section{Figura 6 - Proposta de fluxograma de processo laboratorista}

Com essa nova configuração das atividades, foi proposto a eliminação da busca da impressão, entrega da impressão na recepção e volta até o computador. Tendo um total de 5 operações, 6 transportes e 1 espera, com uma distância percorrida de 34 metros.

Para o processo da recepcionista também foram sugeridas algumas adequações, conforme fluxograma de processo da figura 7.

\begin{tabular}{|c|c|c|c|c|c|c|}
\hline PROCESSO: & \multicolumn{5}{c|}{ Atendimento de pacientes } \\
\hline DESCRIÇÃO DAS ATIVIDADES & $\begin{array}{l}\text { DISTÂNCIA } \\
\text { (metros) }\end{array}$ & \multicolumn{5}{c|}{ PROCESSO } \\
\hline $\begin{array}{l}\text { Atender paciente e acionar alerta de } \\
\text { atendimento para laboratorista, entregar } \\
\text { protocolo ao paciente }\end{array}$ & - & 0 & $\Rightarrow$ & $\square$ & $\square$ & $\square$ \\
\hline TOTAL: & 0 & 1 & 0 & 0 & & \\
\hline
\end{tabular}

\section{Figura 7 - Proposta de fluxograma de processo recepcionista}

Para a operação da recepcionista foi proposta a instalação de um interruptor, para ser acionado quando o paciente estiver pronto para coleta de exames de sangue, esse interruptor aciona uma lâmpada e um sinal sonoro que fica no laboratório, dessa forma o laboratorista sabe que tem paciente para realização de coletas. Também um protocolo com o número do exame que será realizado e entregue ao paciente, no momento do atendimento na chegada ao laboratório. Com isso, a atividade da recepcionista tem 1 operação, não gerando desperdícios.

Para a atividade do paciente foi desenvolvido um fluxograma de processo, de acordo com a figura 8 .

\begin{tabular}{|c|c|c|c|c|c|c|}
\hline PROCESSO: & \multicolumn{6}{|c|}{ Coleta de sangue paciente } \\
\hline DESCRIÇÃO DAS ATIVIDADES & $\begin{array}{c}\text { DISTÂNCIA } \\
\text { (metros) }\end{array}$ & & & PROCESSC & & \\
\hline Chegar no laboratório e ir até recepçăo & 5 & & & & & \\
\hline $\begin{array}{l}\text { Apresentar-se para recepcionista para gerar } \\
\text { protocolo }\end{array}$ & - & & & & & \\
\hline Ir até sala de coleta & 10 & $\mathrm{O}$ & & & & \\
\hline Realizar coleta & - & & & D & & \\
\hline Sair do laboratório & 10 & 0 & & & & \\
\hline TOTAL: & 25 & 2 & 3 & & & \\
\hline
\end{tabular}

Figura 8 - Proposta de fluxograma de processo paciente 
Para o atendimento ao paciente a distância percorrida será de 25 metros, com 2 operações e 3 transportes, foi eliminada a operação de sair da sala de coleta e retirar protocolo, o mesmo será entregue na chegada do paciente.

Um novo layout foi proposto baseado nas informações dos fluxogramas de processo, onde foi possível obter a configuração da figura 9 .

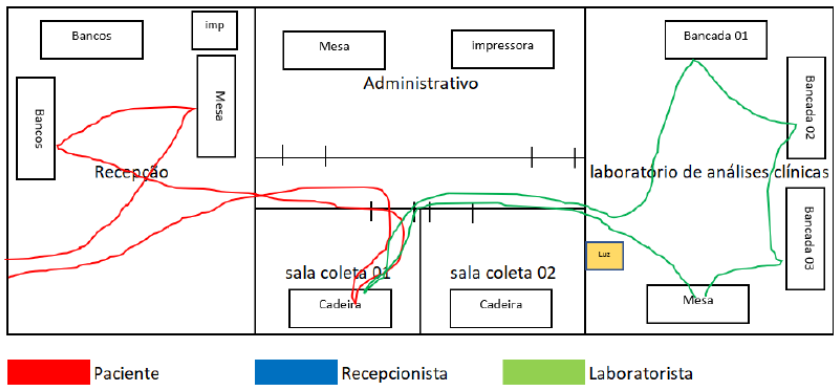

Figura 9 - Proposta de layout para o laboratório

No layout proposto foi elaborado do diagrama de Spaghetti para verificar as movimentações realizadas durante o processo, com essa proposta nota-se que a quantidade de linhas reduziu em relação ao layout anterior, para quantificar essa movimentação e as atividades AV e NAV foi desenvolvida a tabela 2 com os dados.

\begin{tabular}{l|c|c|c}
\hline \multicolumn{1}{c|}{ Atores } & \% Atividades AV & \% Atividades NAV & $\begin{array}{c}\text { Distância } \\
\text { percorrida (metros) }\end{array}$ \\
\hline Laboratorista & $41,6 \%$ & $58,4 \%$ & 34 \\
\hline Recepcionista & $100 \%$ & $0 \%$ & 0 \\
\hline Paciente & $40 \%$ & $60 \%$ & 25 \\
\hline
\end{tabular}

\section{Tabela 2 - Atividades AV, NAV e distâncias percorridas}

Observa-se que a recepcionista ficou isenta de atividades NAV, o laboratorista ficou com $41,6 \%$ de atividades AV e o paciente com $40 \%$.

Para analisar o processo atual com o proposto, serão realizados comparativos de distâncias percorridas, atividades AV e NAV dos processos. Na figura 10 observa-se as distâncias percorridas nas movimentações realizadas pelos atores do processo.

\subsection{Situação Proposta}

Para analisar o processo atual com o proposto, serão realizados comparativos de distâncias percorridas, atividades AV e NAV dos processos. Na figura 10 observa-se as distâncias percorridas nas movimentações realizadas pelos atores do processo.

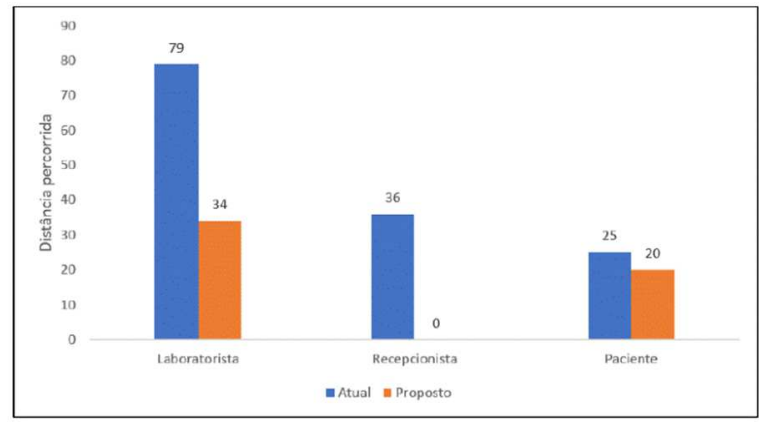

Figura 10 - Comparativo de distâncias percorridas (metros) pelos atores
Com a proposta de layout o laboratorista teve uma redução de 79 para 34 metros, totalizando 45 metros a menos de distância percorrida durante a atividade de coleta de exames de sangue, essa redução foi possível devido a eliminação das atividades de buscar impressão e levar até a recepcionista, para isso foi proposto a instalação de uma impressora ao lado da mesa da recepcionista.

A recepcionista teve uma redução de $100 \%$ nas distâncias percorridas, pois foi eliminada a atividade de chamar laboratorista quando os pacientes estiverem prontos para realização dos exames, isso foi possível graças a proposta de instalação de um interruptor que ficará junto com a mesa da recepcionista, que pode acioná-lo informando o laboratorista com uma luz e sinal sonoro que tem paciente na sala de espera.

Para o paciente houve uma redução de 5 metros de movimentações, pois na situação atual o paciente necessitava retornar a mesa da recepcionista pegar o protocolo de atendimento após o exame, como a proposta o protocolo podera ser entregue na chegada do paciente ao laboratório.

Em relação as atividades que agregam valor (AV) e não agregam valor (NAV) foi realizado um comparativo entre a situação atual e proposta, conforme figura 11.

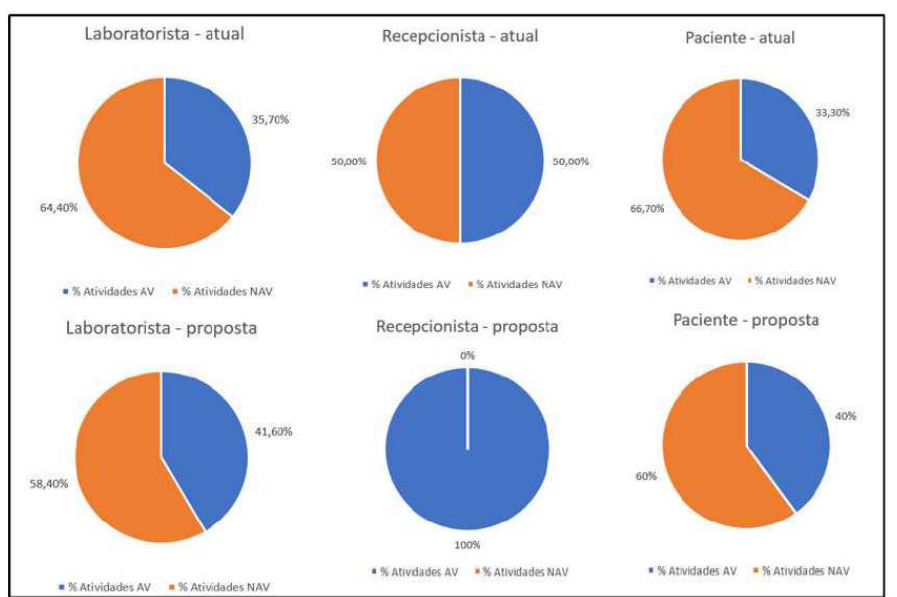

\section{Figura 11 - Comparativo de atividades AV e NAV da situação atual e proposta}

O laboratorista foi de $35,7 \%$ para $41,6 \%$ de AV, a recepcionista de $50 \%$ para $100 \%$ de atividades AV e o paciente de $33,3 \%$ para $40 \%$ de AV. Esses ganhos foram possíveis utilizando as ferramentas de análise Spaghetti e Fluxograma de processo no layout da empresa, e com a participação dos atores do processo dando sugestões de melhorias.

\section{CONSIDERAÇÕES FINAIS}

Ao final deste estudo ficou evidente que um layout mal distribuído pode causar muitos desperdícios no processo de coleta de exames de sangue, estes desperdícios aumentam as movimentações e transportes durante a execução das atividades, causando ineficiências no processo.

Através do fluxograma de processo e diagrama de Spaghetti foi possível realizar o mapeamento do processo dos atores envolvidos nas atividades e seus respectivos deslocamentos durante as etapas, também a classificação das atividades que agregam valor (AV) e não agregam valor (NAV). Após o uso dessas ferramentas foi possível reunir os envolvidos no processo 
para propor melhorias no layout e na realização das atividades, através disso, foi elaborada uma proposta de layout onde houveram reduções de movimentações, no caso do laboratorista foram reduzidos 45 metros de deslocamento para coleta de exames de sangue.

Para reduzir movimentações do laboratorista e recepcionista foram propostas a utilização de sinalizadores luminosos e sonoros para informar que os pacientes estão prontos para realização de exames, também a instalação de uma impressora para a recepcionista para eliminar a movimentação do laboratorista na impressão de exames.

Foi verificado que o fluxograma de processo e diagrama de spaghetti são ferramentas que podem trazer ótimos resultados para as empresas, independente do porte ou atividades, pois e possível visualizar todos os atores envolvidos, atividades realizadas e suas movimentações durante os processos.

\section{R E F E R E N C E S}

BARNES, Ralph Mosser. Estudo de movimentos e de tempos: projeto e medida do trabalho. Editora Edgard Blucher, 1977.

CAMAROTTO, J. A.; MENEGON N. L. Projeto de Unidades Produtivas: Apostila. São Carlos: Departamento de Engenharia de Produção, Centro de Ciências Exatas e de Tecnologia, Universidade Federal de São Carlos, 2006.

CORRÊA, H. L., GIANESI, 1. G., \& CAON, M. (2001). Planejamento, programação e controle da produção. São Paulo: Atlas, 1.

JOHANSSON, H. J. Processos de negócios. São Paulo: Pioneira, 1995

MARIZ, R. N., \& PICCHI, F. A. (2013). Método para aplicação do trabalho padronizado. Ambiente Construído.

OHNO, T. Sistema Toyota de Produção - Além da Produção em Larga Escala. Porto Alegre: Bookman, 1997.

PEINADO, J., \& GRAEML, A. R. (2007). Administração da produção. Operações industriais e de serviços. Unicenp.

SHINGO, S. O Sistema Toyota de Produção do ponto de vista da engenharia de produção. Porto Alegre: Bookman, 1996.

SHINGO, Shigeo. The Toyota production system. Tokyo: Japan Management Association, 1981.

SLACK, N., CHAMBERS, S., \& JOHNSTON, R. (2009). Administração da produção (Vol. 747). São Paulo: Atlas.

VIEIRA, Everton Luiz. Signatures factory: a dynamic alternative for teaching-learning layout concepts and waste disposal. Production, v. 27, n. SPE, 2017. http://dx.doi.org/10.1590/0103-6513.221716

WOMACK, J. P.; JONES, D. T. A Mentalidade enxuta nas empresas: elimine o desperdício e crie riquezas. Rio de Janeiro: Campus, 1998. 